\title{
A Case of Lymphomatoid Keratosis
}

\author{
Min Jee Choi, M.D., Hei Sung Kim, M.D., Hyung Ok Kim, M.D., Kye Yong Song, M.D. ${ }^{1}$, \\ Young Min Park, M.D.
}

Department of Dermatology, Seoul St. Mary's Hospital, College of Medicine, The Catholic University of Korea, ${ }^{1}$ Department of Pathology, College of Medicine, Chung-Ang University, Seoul, Korea

Lymphomatoid keratosis (LK) is considered to be a rare variant of cutaneous lymphoid hyperplasia, with epidermotropism. We herein report a case of LK which developed on the abdomen of an elderly Korean woman. A 60-year-old woman presented with a 10-year history of a pruritic, solitary, brown to black plaque on the abdomen. Histopathologically, the specimen showed hyperkeratosis, parakeratosis, acanthosis and Pautrier's micro-abscess in the epidermis, and a lichenoid infiltration of lymphocytes in the dermis, which expressed both B cell and T cell lineage on the immune-histochemical staining. Based on these clinical and histopathological findings, our case was diagnosed as LK. To our knowledge, this is the first case report of LK in the Korean dermatologic literature. (Ann Dermatol 22(2) 219 222, 2010)

\section{-Keywords-}

Cutaneous lymphoid hyperplasia, Epidermotropism, Lymphomatoid keratosis

\section{INTRODUCTION}

Lymphomatoid keratosis (LK) has been considered to be a variant of uni-lesional mycosis fungoides (MF), as it shows epidermotropism that is characterstic of $\mathrm{MF}^{1}$. With its lichenoid keratotic features, it also has been recognized as

Received February 2, 2009, Revised June 10, 2009, Accepted for publication September 1, 2009

*This study was supported by a grant of the Korea Healthcare technology R\&D Project. Ministry for Health, Welfare \& Family Affairs, Republic of Korea (A090084).

Corresponding author: Young Min Park, M.D., Department of Dermatology, Seoul St. Mary's Hospital, College of Medicine, The Catholic University of Korea, 505 Banpo-dong, Seocho-gu, Seoul 137-701, Korea. Tel: 82-2-2258-6223, Fax: 82-2-594-3255, E-mail: yymmpark6301@ hotmail.com a subtype of benign lichenoid keratosis ${ }^{2}$. Recently, Arai et al. ${ }^{3}$ proposed that LK is a epidermotropic type of cutaneous lymphoid hyperplasia, where differentiation from MF and benign lichenoid keratosis is possible, by comparing the clinocopathological, immunohistochemical and molecular biological findings. To date, there have been 26 case reports of LK in the English literature, but none in the Korean dermatologic literature. Herein, we report the first case of LK in Korea and review the previous cases.

\section{CASE REPORT}

A 60-year-old woman presented with a 10-year history of a pruritic, solitary, well-demarcated, scaly, brown to black plaque on the abdomen, measuring $3.8 \times 1.2 \mathrm{~cm}$ in size, which had started as a tiny papule (Fig. 1). Dermoscopic findings revealed numerous brown dots and globules at the periphery (Fig. 2). She had no previous personal or familial history of skin cancer and no other significant cutaneous or medical history was found. There was no history of trauma. A skin biopsy from a local clinic 4 years before showed hyperkeratosis, parakeratosis, acanthosis, papillomatosis and hypergranulosis in the epidermis, which was consistent with seborrheic keratosis. Additio-

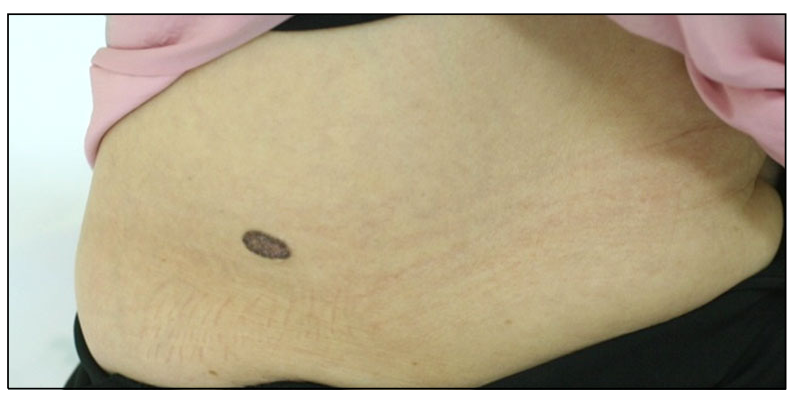

Fig. 1. Pruritic, solitary, well-demarcated, brown to black plaque, $3.8 \times 1.2 \mathrm{~cm}$ in size, on the abdomen. 


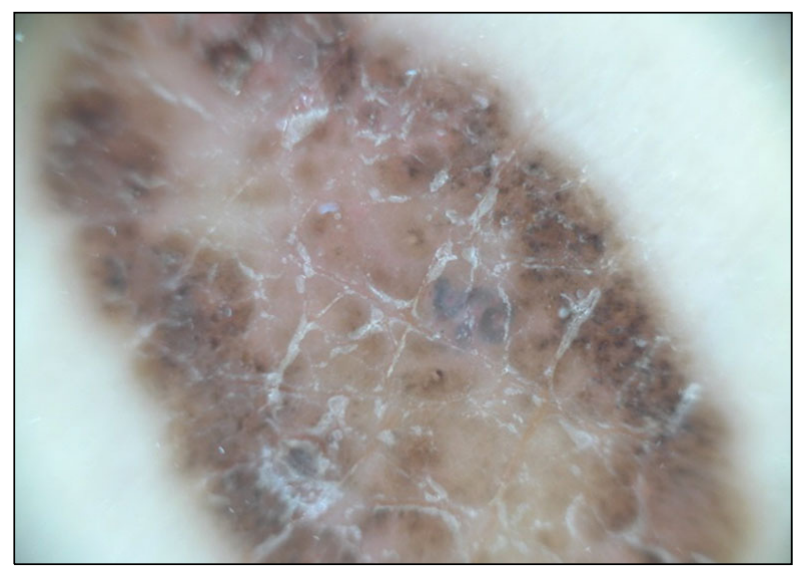

Fig. 2. Dermoscopic finding of the lesion showed a globular pattern with brown dots and globules at the periphery.

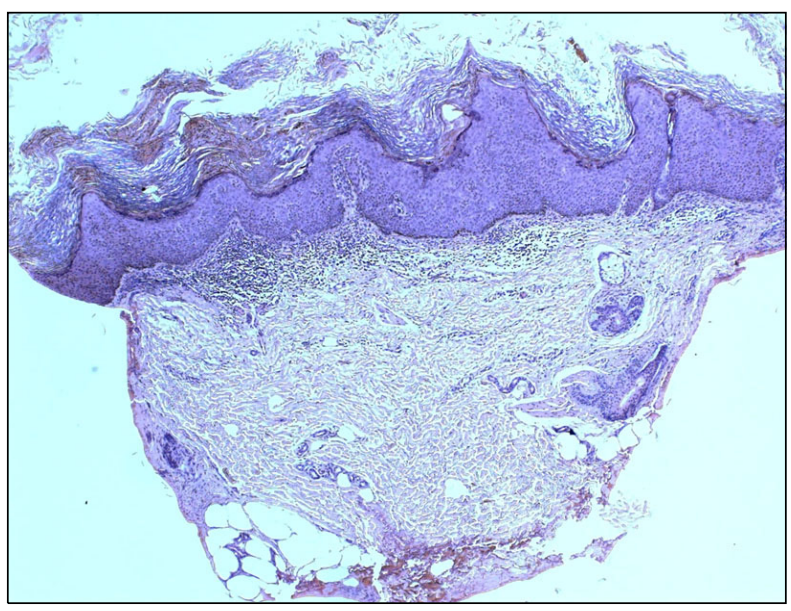

Fig. 3. Hyperkeratosis, parakeratosis, acanthosis, papillomatosis and hypergranulosis in the epidermis which was consistent with seborrheic keratosis $(\mathrm{H} \& \mathrm{E}, \times 40)$.

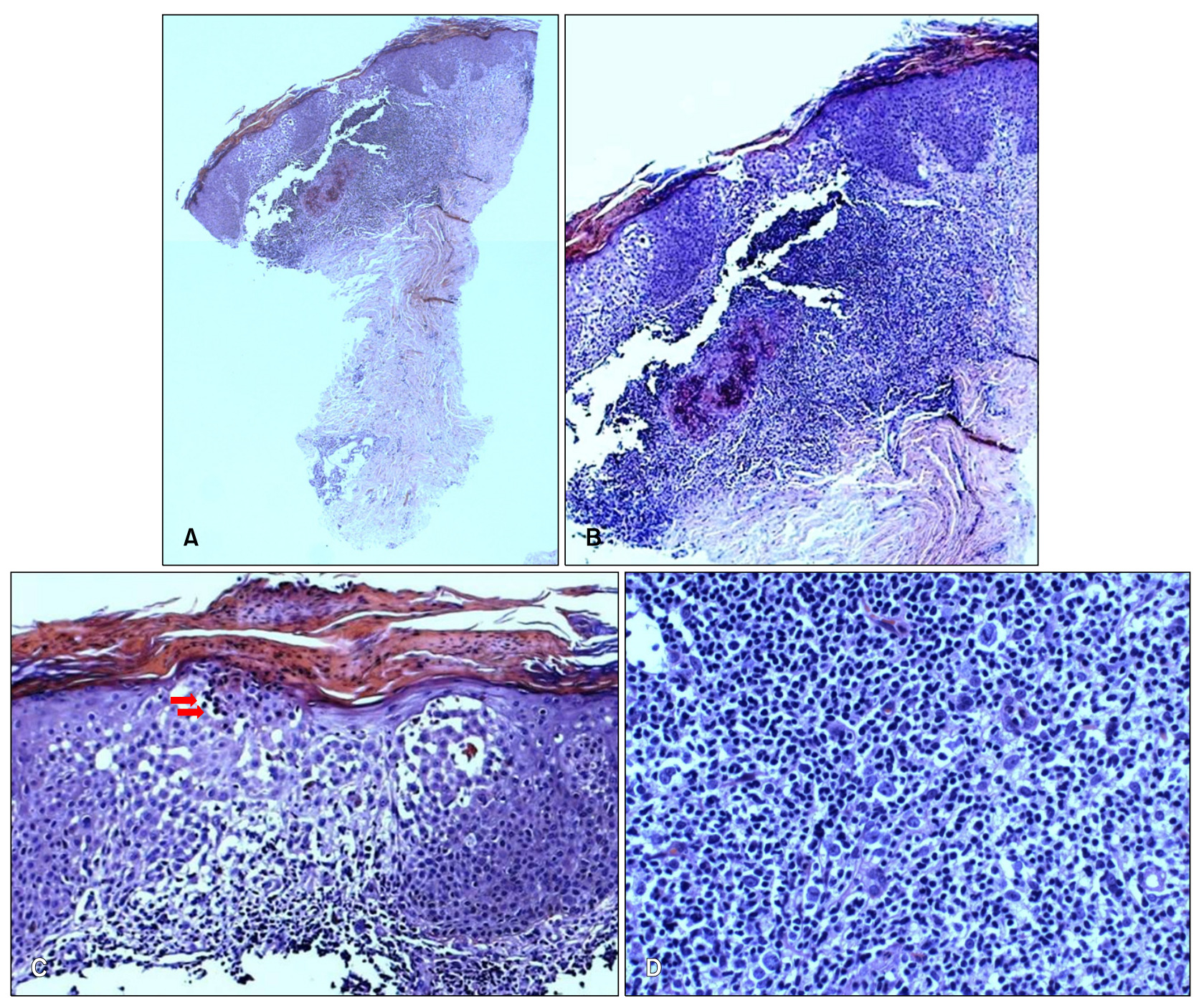

Fig. 4. (A, B) Epidermal hyperplasia and epidermotropism in the epidermis and formation of lymphoid follicle and lichenoid infiltration of lymphocytes in the reticular dermis ( $\mathrm{H} \& \mathrm{E}$ stain, $\times 40, \times 100)$. (C) Epidermotropism with Pautrier's microabscess in the epidermis (H\&E stain, $\times 400)$. (D) Lichenoid infiltration of lymphocytes in the reticular dermis (H\&E stain, $\times 400)$. 

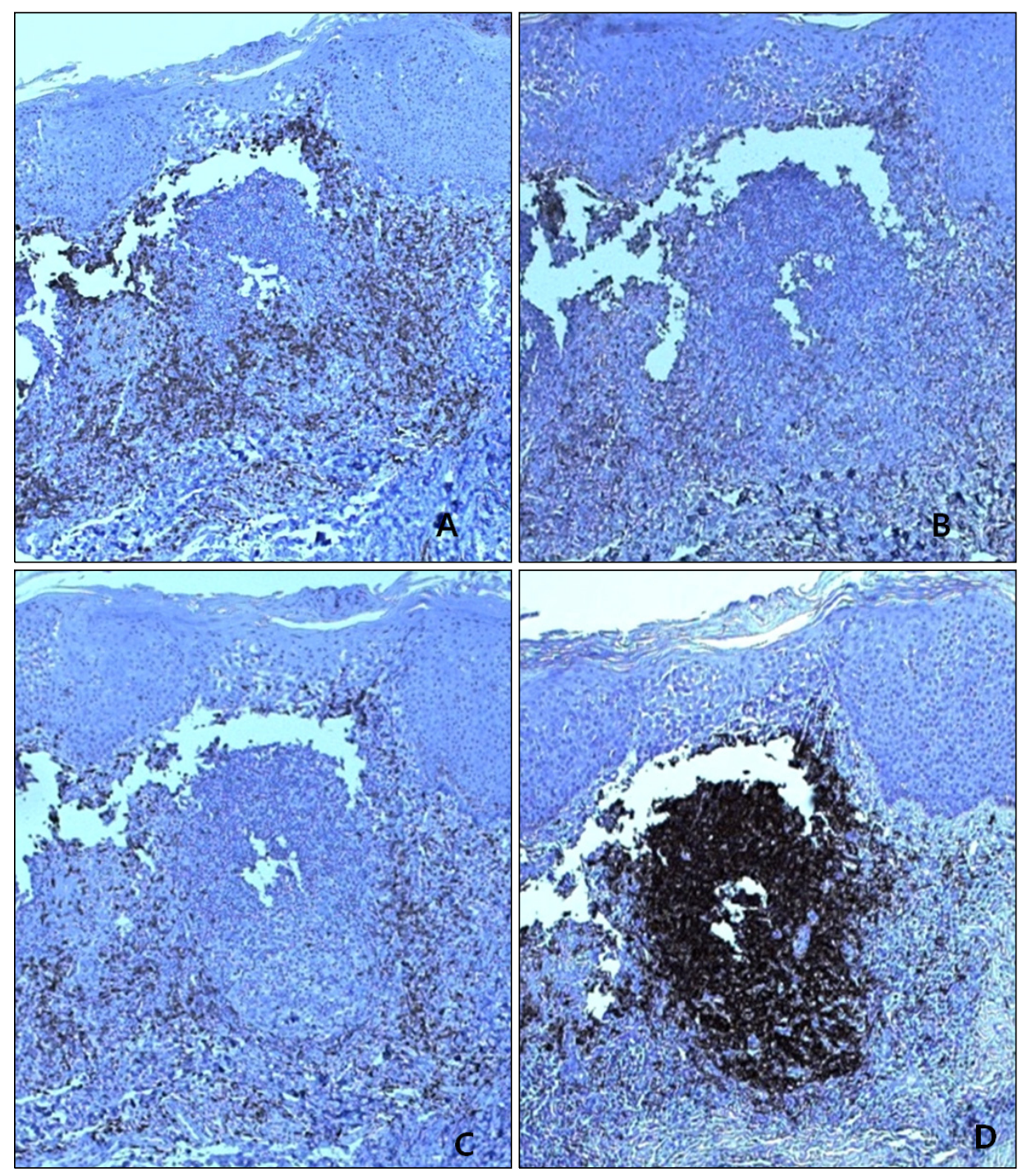

Fig. 5. Immunohistochemical study for CD3 (A), CD4 (B), CD8 (C), CD20 (D), showed positive staining with lymphocytes $(\mathrm{H} \& \mathrm{E}, \times 400)$. nally, a dense infiltration of lymphocytes in the superficial dermis was observed (Fig. 3).

To confirm the previous diagnosis and rule out malignancy, we performed a second skin biopsy from the same lesion. Histopathological findings showed hyperkeratosis, parakeratosis, acanthosis and epidermotropism with Pautrier's micro-abscess in the epidermis, a lichenoid infiltration of lymphocytes, the formation of lymphoid follicle and hemorrhage in the reticular dermis (Fig. 4). Solar elastosis was not seen. Immuno-histopathologically, the exocytic lymphocytes in the epidermis and the lymphocytes of the lymphoid follicle in the dermis were positive for CD3, CD4, CD8, CD20, CD30 and CD79a (Fig. 5). Genotypically, rearrangement of TCR $\gamma$ was partially demonstrated. Based on these clinical and histopathological findings, we made a final diagnosis of LK. Complete excision of the lesion was performed. No local recurrence occurred during the ensuing 3 months.

\section{DISCUSSION}

Evans et al. ${ }^{1}$ first reported a case of uni-lesional MF on the right flank area of a 44-year-old male, where the lesion demonstrated histopathological features of epidermotropism with lichenoid activity. Later on, Kossard ${ }^{2}$ suggested that the case reported by Evans et al. ${ }^{1}$ should have been categorized as LK, thereby introducing the term LK for the first time in 1997. Thereafter, there have been 23 cases of LK reported in the English literature.

LK clinically presents as an asymptomatic, scaly, erythematous plaque on the face or upper trunk of the middle aged personnel, simulating basal cell carcinoma, actinic keratosis, or seborrheic keratosis ${ }^{2,4,5}$. Most cases of LK are idiopathic, however several have been associated with 
newly encountered antigens from anthropod bites, stings, tattoo, vaccinations, trauma, injection of foreign substances, pierced ear jewelry, and drugs ${ }^{6}$. Al-Hoqail and Crawford $^{4}$ reported cases of LK adjacent to seborrheic keratosis and solar lentigo. Coexistence with these diseases make us suspect that LK might be related to solar damage. In our case, though, solar elastosis was not observed in the dermis. Further evaluation is needed to explain the clear relationship between LK and solar damage. The most pathognomic pathological finding of LK is epidermotropism. Lymphocytes with tropism for the epidermis are composed of both B cells and T cells ${ }^{3}$.

Special attention is needed to differentiate LK from uni-lesional MF because these 2 diseases largely resemble one another. Uni-lesional MF is a rare variant of MF which usually appears as a single, isolated lesion comprising of less than $5 \%$ of the body's surface area. The disease is usually benign and has an excellent response to locally ablative treatments ${ }^{7}$. Uni-lesional MF is different from LK in that the epidermotropic lymphocytes in uni-lesional MF mainly consist of helper T cells possessing T cell clonality, which conveys the malignant transformation ${ }^{3}$.

Other major differentials to consider are lichenoid actinic keratosis and benign lichenoid keratosis. Both of them commonly show lichenoid infiltrates of lymphocytes with epidermal hyperplasia. However, lichenoid actinic keratosis differs from LK in that atypical basal cells and keratinocytes can be found whereas epidermotropism of the lymphocytes are not seen. Benign lichenoid keratosis shows necrotic keratinocytes, spongiosis and Max-Joseph spaces without epidermotropism ${ }^{8}$. Also, irritated seborrheic keratosis and inflamed seborrheic keratosis should be included in the differential diagnosis of LK in that the biopsy specimen from the same lesions 4 years earlier was consistent with seborrheic keratosis. Irritated seborrheic keratosis differs from LK in that in the former, squamous cell eddies or pearls can be found, and epidermotropism of the lymphocytes are not seen (even though inflammation is severe). Inflamed seborrheic keratosis differs from LK in that spongiosis and exocytosis of lymphocytes in the epidermis can be found in the former, and epidermotropism of the lymphocytes are not seen ${ }^{9,10}$.

Based on the histopathological and immunohistochemical findings, our case was diagnosed as LK. The unusual feature in our case is the history of a transformation from seborrheic keratosis to LK. Either the transformation of se- borrheic keratosis to LK is incidental or else these two diseases may be truly related. Chronic rubbing may induce hyperkeratotic changes in the epidermis and exocytosis and spongiosis of lymphocytes in the dermis, reflecting the unidentified culprit factor of LK like other spongiotic dermatitis. For a clear explanation, further investigation is needed.

In conclusion, we experienced a typical case of LK that developed on the abdomen and was transformed from seborrheic keratosis. LK is an epidermotropic kind of cutaneous lymphoid hyperplasia, with hyperkeratotic changes in the epidermis, therefore it should be included in the differential diagnosis of benign keratotic dermatosis.

\section{REFERENCES}

1. Evans LT, Mackey SL, Vidmar DA. An asymptomatic scaly plaque. Unilesional mycosis fungoides (MF). Arch Dermatol 1997;133:231, 234.

2. Kossard S. Unilesional mycosis fungoides or lymphomatoid keratosis? Arch Dermatol 1997;133:1312-1313.

3. Arai E, Shimizu M, Tsuchida T, Izaki S, Ogawa F, Hirose T. Lymphomatoid keratosis: an epidermotropic type of cutaneous lymphoid hyperplasia: clinicopathological, immunohistochemical, and molecular biological study of 6 cases. Arch Dermatol 2007;143:53-59.

4. Al-Hoqail IA, Crawford RI. Benign lichenoid keratoses with histologic features of mycosis fungoides: clinicopathologic description of a clinically significant histologic pattern. J Cutan Pathol 2002;29:291-294.

5. Lever WF, Schaumburg-Lever G. Histopathology of the skin. 6th ed. London: Lippincott, 1983:762-768.

6. Bergman R, Khamaysi Z, Sahar D, Ben-Arieh Y. Cutaneous lymphoid hyperplasia presenting as a solitary facial nodule: clinical, histopathological, immunophenotypical, and molecular studies. Arch Dermatol 2006;142:1561-1566.

7. Heald PW, Glusac EJ. Unilesional cutaneous T-cell lymphoma: clinical features, therapy, and follow-up of 10 patients with a treatment-responsive mycosis fungoides variant. J Am Acad Dermatol 2000;42:283-285.

8. Murphy GF, Schwarting R. Cutaneous lymphomas and leukemias. In: Elder DE, Elenitsas R, Johnson BL Jr, Murphy GF, editors. Lever's histopathology of the skin. 9th ed. Philadelphia: Lippincott Williams \& Wilkins, 2005:927-978.

9. Bazza MA, Ryatt KS, Dharmagunawardena PV. Mycosis fungoides masquerading as seborrhoeic keratosis. $\mathrm{Br}$ J Dermatol 2002;147:1264-1265.

10. Yoo SS, Viglione $M$, Moresi $M$, Vonderheid E. Unilesional mycosis fungoides mimicking Bowen's disease. J Dermatol 2003;30:417-419. 